\title{
Stability of Sampled-Data, Delayed Haptic Interaction and Teleoperation
}

\author{
Noushin Miandashti*
}

Mahdi Tavakoli, IEEE Member ${ }^{\dagger}$

Department of Electrical and Computer Engineering, University of Alberta, Edmonton, AB T6G 2V4, Canada

\begin{abstract}
This paper proposes a unified framework to study the stability of sampled-data, haptic virtual environment (HVE) systems and sampled-data position-error-based (PEB) bilateral teleoperation systems based on the discrete-time circle criterion. Communication time delay and controller discretization are two major factors that jeopardize the system stability. We provide a framework for the system stability analysis in which both these two destabilizing factors can be addressed. In this paper, first the well-known Colgates stability condition for a 1-user haptic system with a passive operator is reproduced in a different manner and then extended to the case where delay can exist in the communication channel. Then, it is shown that using the same method, the stability of sampled-data position-error-based (PEB) bilateral teleoperation systems can be dealt in a similar manner. Simulation results confirm the validity of the proposed conditions for stability of both sampled-data, HVE and PEB bilateral teleoperation systems.
\end{abstract}

Keywords: HVE systems, PEB bilateral teleoperation systems, circle criterion.

Index Terms: H.5.2 [Information Interfaces and Presentation]: User Interfaces-Haptic I/O; I.2.9 [Artificial Intelligence]: Problem Solving, Control Methods, and Search-Control theory

\section{INTRODUCTION}

Many applications of haptic enabled interfaces can fall into two main categories: Haptic virtual environment (HVE) systems, and teleoperation systems. An HVE system consists of a human operator and a virtual environment and a haptic interface that acts as a link between them and conveys a kinesthetic sense of presence in the virtual environment to the operator. Surgical simulation $[14,1]$ and telerehabilitation [3] are only a few applications of HVE systems. A teleoperation system comprises of a human operator interacting with a master robot, thus remotely controlling a slave robot to perform a task in a remote environment. Ideally, from a performance perspective, the slave robot exactly reproduces the master's position trajectory while the master robot reproduces the slaveenvironment contact force for the human operator; this is called bilateral teleoperation. Bilateral teleoperation has applications including telesurgery and remote underwater and space exploration. Surveys on bilateral teleoperation can be found in [12, 13]. A number of authors have considered the issue of stability in sampleddata haptic interaction in the virtual space. A more rigorous examination of stability was performed by Colgate and Schenkel [4]. For a common discrete-time implementation of the virtual environment composed of a spring and a damper in parallel, the necessary and sufficient condition for passivity and absolute stability of the

*e-mail: miandash@ualberta.ca

†e-mail: mahdi.tavakoli@ualberta.ca

IEEE Haptics Symposium 2014

23-26 February, Houston, Tx, USA

978-1-4799-3130-9/14/\$31.00 @2014 IEEE sampled-data HVE system was derived as

$$
b>\frac{K T}{2}+|B|
$$

Another approach to the stability analysis of a similar HVE system was provided by Gil et al. In a series of papers [5, 8], using the Routh-Hurwitz criterion, the closed-loop stability problem of the 1-DOF, HVE system was addressed directly. The environment was modeled as a virtual spring and damper in mechanical parallel and the stability condition for a delayed HVE system, which delay $t_{d}$ can be the sum of several effects (computations, communications, etc), the stability condition was found to be

$$
K<\frac{B+b}{\frac{T}{2}+t_{d}}
$$

The above condition was shown to be valid only for low values of the virtual damping B. In the context of bilateral teleoperation, in more recent studies, the effects of discretized controllers on system passivity and stability have been investigated $[9,10]$. In this paper, a discrete-time circle criterion based framework to find the stability condition for a sampled-data, delayed haptic system as well as a sampled-data delayed bilateral teleoperation system under PEB architecture is proposed. The rest of the paper is organized as follows. Section 2 provides mathematical preliminaries required for the rest of the paper. In Section 3, for the haptic system in Figure 2a, stability conditions are derived for both under delayed or non-delayed channels. Section 4, studies the stability of a sampled-data PEB bilateral teleoperation system under delayed or non-delayed channels. Simulation results are presented in Section 5 and Section 6 presents the conclusions.

\section{Mathematical Preliminaries}

In this section mathematical preliminaries required for the rest of the paper are provided:

Lemma 1. [11] The LTI minimal realization

$$
\begin{array}{r}
x(i+1)=A x(i)+B u(i) \\
y(i)=C x(i)+D u(i)
\end{array}
$$

with $G(z)=C(z I-A)^{-1} B+D$ is

- passive if $G(z)$ is positive real;

- strictly passive if $G(z)$ is strictly positive real.

Definition 1. [11] An $m \times m$ proper rational transfer function $m a-$ trix $G(z)$ is positive real if

- poles of all elements of $G(z)$ are inside or on the unit circle

- for all real $\omega$ for which $e^{j \omega}$ is not a pole of any element of $G(z)$, the matrix $G\left(e^{j \omega}\right)+G^{T}\left(e^{-j \omega}\right)$ is positive semidefinite, and 


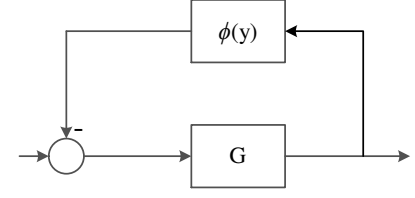

Figure 1: The model of a sampled-data feedback system with LTI system $G$ in the forward path and the nonlinearity $\phi=\phi(y)$ in the feedback path

- the poles of any element of $G(z)$ on $|z|=1$ are simple and the associated residue matrices of these poles are positive semidefinite.

Definition 2. [11] Let A be a Hermitian symmetric matrix. A is positive semidefinite, if all its leading principle minors are nonnegative. We say that $A$ is positive definite if all its leading principle minors are positive.

Theorem 2.1. [7] Consider a sampled-data multivariable control system that consists of an LTI system in the forward path and the nonlinearity $\phi=\phi(y)$ in the feedback path as shown in Figure 1. Such a system can be presented by the difference equation

$$
\begin{aligned}
& x(i+1)=A x(i)-B \phi(y) \\
& y(i)=C x(i), y \in R^{m} \\
& \phi(y)=\left[\phi_{1}\left(y_{1}\right), \phi_{2}\left(y_{2}\right), \ldots, \phi_{m}\left(y_{m}\right)\right]^{T}
\end{aligned}
$$

If there exists $K=\operatorname{diag}\left(k_{1}, \ldots, k_{m}\right)>0$ such that

$$
K^{-1}+C(z I-A)^{-1} B
$$

is positive real then $G(z)$ is absolutely stable for any $\phi$ satisfying

$$
\phi(0)=0,0<y_{i} \phi\left(y_{i}\right) \leq y_{i}^{2} k_{i}
$$

Thus, the sampled-data system (5)-(7) will be stable. For a passive $\phi, k_{i} \rightarrow \infty$ and condition (8) will change to $G(z)$ being positive real.

\section{Stability analysis of a Sampled-Data HVE Sys- TEM}

The block diagram of the HVE system is shown in Figure 2a, where $Z_{h}(s)$ is the unknown human operator model and $H(z)$ is the known discrete-time model of the environment (i.e., the digitallyimplemented virtual coupling between the haptic interface and the virtual wall). The haptic interface is a rigid manipulator and is modeled as a mass $m$ and a damper $b$. The input and output of $H(z)$ pass through a sampler and a $\mathrm{ZOH}$ with a sampling period of $T$, respectively. Simple manipulations in the block diagram in Figure $2 \mathrm{a}$ will result in the one in Figure $2 b$. The equations governing the resulting system in Figure $2 b$ will be

$$
\begin{array}{r}
f_{h}-u=b v_{h} \\
x_{h}=\frac{v_{h}}{s} \\
u^{*}=z^{-n} H(z) x_{h}^{*}
\end{array}
$$

With the assumption that $n=t_{d} / T$ is an integer ( $t_{d}$ represents the communication delay), the discrete-time equivalent of (10) is

$$
f_{h}^{*}-u^{*}=b v_{h}^{*}
$$

and with the help of (12) we get

$$
f^{*}-z^{-n} H(z) x_{h}^{*}=b v_{h}^{*}
$$

The above can be written in the $z$-domain as

$$
F(z)-z^{-n} H(z) X_{h}(z)=b V_{h}(z)
$$

where

$$
X_{h}(z)=\mathscr{Z}\left\{\frac{v_{h}}{s}\right\}
$$

It is important to note that $\mathscr{Z}\left\{\frac{v_{h}}{s}\right\} \neq \mathscr{Z}\left\{\frac{1}{s}\right\} V_{h}(z)$. To be able to

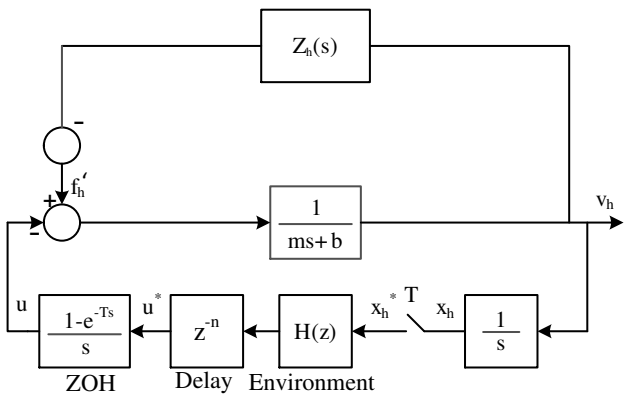

(a)

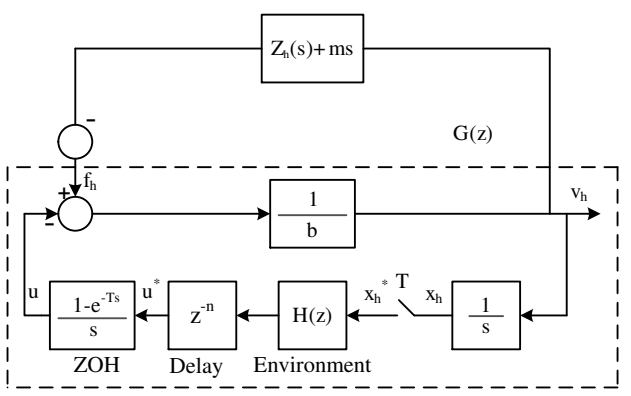

(b)

Figure 2: Model of a 1-DoF sampled-data HVE system

derive the transfer function from $f_{h}$ to $v_{h}$, we need to approximate $\mathscr{Z}\left\{\frac{v_{h}}{s}\right\}$. We can do so based on one of the following approximation methods:

- Forward Difference: $\quad X_{h}(z)=\frac{T}{z-1} V_{h}(z)$

- Backward Difference: $\quad X_{h}(z)=\frac{T z}{z-1} V_{h}(z)$

- Tustins Transformation: $X_{h}(z)=\frac{T}{2} \frac{z+1}{z-1} V_{h}(z)$

In previous related works $[4,8,6]$ the impedance of the environment in the $z$ domain has been assumed as $H(z)=K+\frac{B(z-1)}{T z}$ and, we will use the same model. In the following, we consider two cases for a passive operator.

Passive Operator, No Delay Assuming that $t_{d}=0$, depending on which approximation is chosen, the $f$ to $v$ mapping will be one of the following:

$$
\begin{gathered}
F_{h}(z)=b V_{h}(z)+\left(K+\frac{B(z-1)}{T z}\right) \frac{T}{z-1} V_{h}(z)=G_{1}^{-1}(z) V_{h}(z) \\
F_{h}(z)=b V_{h}(z)+\left(K+\frac{B(z-1)}{T z}\right) \frac{T z}{z-1} V_{h}(z)=G_{2}^{-1}(z) V_{h}(z) \\
F_{h}(z)=b V_{h}(z)+\left(K+\frac{B(z-1)}{T z}\right) \frac{T}{2} \frac{z+1}{z-1} V_{h}(z)=G_{3}^{-1}(z) V_{h}(z)
\end{gathered}
$$

The above correspond to forward difference, backward difference and Tustin approximations, respectively. Based on Theorem 2.1 


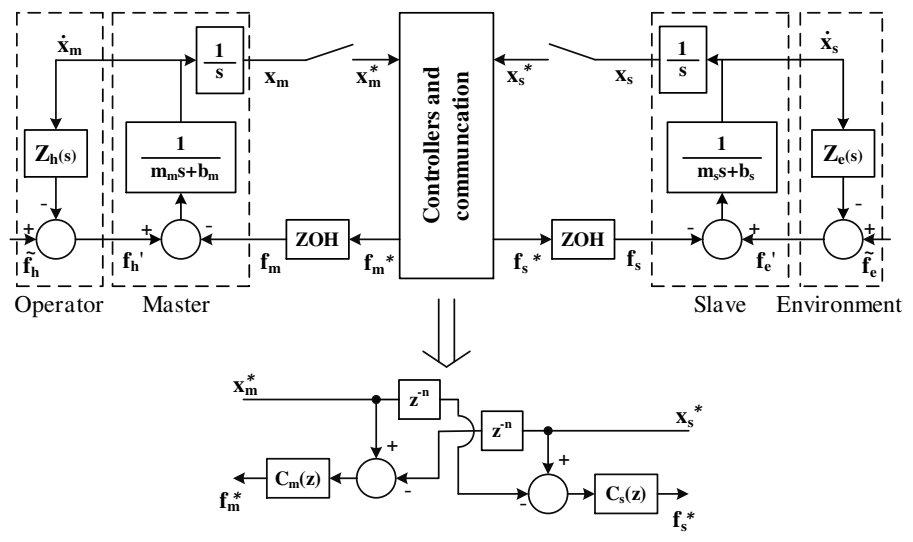

Figure 3: Model of a sampled-data bilateral teleoperation system

since $Z_{h}(s)+m s$ is passive, the system in Figure $2 \mathrm{~b}$ is stable if $G(z)$ is positive real. Based on Lemma 1 and using the fact that passivity of $G(z)$ is equal to $G^{-1}(z)$ being passive [2], the stability of the system is ensured if $G^{-1}(z)$ is positive real. The first condition for positive realness of $G^{-1}(z)$ is satisfied since it can be clearly seen that the poles of $G_{i}^{-1}(z), i=1,2,3$, lie inside or on the unit circle. Since $z=1$ is a simple pole and its residue for each $G_{i}^{-1}(z)$ is positive semidefinite, $K T$, the only remaining condition to check is the second condition in the Definition 1, which requires $\Re\left\{G_{i}^{-1}(z)\right\} \geq 0$. In this way, the conditions for stability of the HVE system based on the three approximations can be found. It can be easily shown that the forward difference approximation method results in the worst-case condition and as a result in the rest of this paper the $\frac{1}{s}$ is approximated using the forward difference approximation method. Using forward difference method the condition for stability of a non-delayed HVE system is

$$
b>\frac{K T}{2}+B
$$

which is identical to Colgate's condition.

Passive Operator, Delay The previous condition was found assuming no delay in the system. For a delayed HVE system, the stability condition will take a different form depending on the virtual environment model. Here again, it can be shown that forward difference will lead us to the worst-case condition. The $f$ to $v$ mapping in the $z$ domain will then be

$$
F_{h}(z)=b V_{h}(z)+z^{-n}\left(K+\frac{B(z-1)}{T z}\right) \frac{T}{z-1} V_{h}(z)
$$

Since the passivity of $G(z)$ is equal to $G^{-1}(z)$ being passive, the delayed sampled-data HVE system is stable if (15) is positive real:

$$
G^{-1}(z)=b+z^{-n}\left(K+\frac{B(z-1)}{T z}\right) \frac{T}{z-1}
$$

The first and third conditions in Definition 1 are obviously the case here since all poles of $G(z)^{-1}$ are on or inside the unit circle and the $z=1$ is a simple pole with positive residue. This will leave us with the third condition which requires $\mathfrak{R}\left\{G^{-1}(z)\right\} \geq 0$. Substituting $z=\cos (\omega T)+j \sin (\omega T)$ in (15) the real part of $G(j \omega)^{-1}$ must satisfy:

$$
b+B \cos \left(\omega t_{d}-T\right)-\frac{K T}{2} \cos \left(\omega t_{d}\right)-K T S>0
$$

where, $S=\frac{\sin \left(\omega t_{d}\right) \sin (\omega T)}{2(1-\cos (\omega T))}$. With the assumption that $t_{d} / T=n$ and $B$ is small, the worst-case condition will happen if $\cos (\omega T)=1$.Then, it can be easily shown that (16) will simplify to

$$
b+B-\frac{K T}{2}-K t_{d} \cos (\omega T / 2)>0
$$

For $\cos (\omega T / 2)=1$ the passivity condition for a delayed HVE system will be derived as follow:

$$
b+B>\frac{K T}{2}+K t_{d}
$$

Interestingly, the above condition is identical to the condition reported in [6].

\section{Stability Analysis of PEB Bilateral Teleopera- TION SYSTEMS}

A sampled-data teleoperation system with PEB architecture is modeled in Figure 3. The master and slave robots are modeled as 1DOF, mass-damper, LTI systems with the following dynamics:

$$
\begin{array}{r}
f_{h}^{\prime}-f_{m}=m_{m} \ddot{x}_{m}+b_{m} \dot{x}_{m} \\
f_{e}^{\prime}-f_{s}=m_{s} \ddot{x}_{s}+b_{s} \dot{x}_{s}
\end{array}
$$

In the above, $f_{h}^{\prime}$ and $f_{e}^{\prime}$ are the human operator and the environment forces. Also, $f_{m}$ and $f_{s}$ are the control actions from the master and the slave discrete-time controllers $C_{m}(z)$ and $C_{s}(z)$. The parameters $b_{m}$ and $b_{s}$ are the dampings and $m_{m}$ and $m_{s}$ are the masses of the master and slave robots, respectively. In the force-velocity domain, the operator and the environment are modeled as LTI impedances $Z_{h}(s)$ and $Z_{e}(s)$, respectively. In Figure $3, \tilde{f}_{h}$ is the exogenous input force from the operator and $\tilde{f}_{e}$ is the exogenous input force from the environment. As shown in Figure 3, the positions of the master and robots are discretized using sampler blocks. The superscript ${ }^{*}$ denotes sampled signals. $\mathrm{ZOH}$ blocks convert the sampled signals back to the continuous-time domain. The PEB controller blocks in Figure 3 apply forces based on the master/slave position difference while considering the time delay incurred when each position signal travels to the other end of the teleoperation system. Thus, the discrete-time controllers of the master and the slave implement the following laws:

$$
\begin{array}{r}
F_{m}^{*}(s)=\left.C_{m}(z)\right|_{z=e^{s T}}\left[X_{m}^{*}(s)-z^{-n} X_{s}^{*}(s)\right] \\
F_{s}^{*}(s)=\left.C_{s}(z)\right|_{z=e^{s T}}\left[X_{s}^{*}(s)-z^{-n} X_{m}^{*}(s)\right]
\end{array}
$$




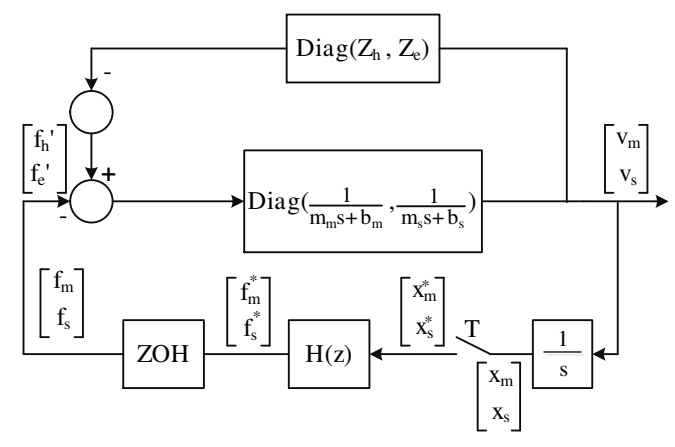

(a)

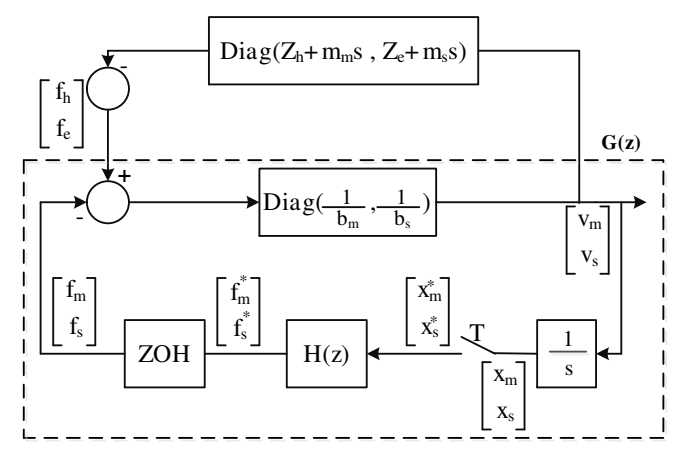

(b)

Figure 4: Block diagram of a teleoperation system, which includes discretized controller models

The system in Figure 3 can be represented as the block diagram in Figure 4 a where delay $n=\frac{t_{d}}{T}$ is an integer and

$$
H(z)=\left[\begin{array}{cc}
C_{m}(z) & -z^{-n} C_{m}(z) \\
-z^{-n} C_{s}(z) & C_{s}(z)
\end{array}\right]
$$

Simple manipulation in Figure $4 \mathrm{a}$ will result in Figure $4 \mathrm{~b}$. Note that by moving the masses of the master and slave robots $m_{m}$ and $m_{s}$ to the operator and environment impedances, the closed-loop transfer matrix will not change. Then, the mapping for the system in Figure $4 \mathrm{~b}$ can be written as:

$$
\left[\begin{array}{l}
F_{h}(z) \\
F_{e}(z)
\end{array}\right]=\left[\begin{array}{cc}
b_{m} & 0 \\
0 & b_{s}
\end{array}\right]\left[\begin{array}{l}
V_{m}(z) \\
V_{s}(z)
\end{array}\right]+H(z) \mathscr{Z}\left[\begin{array}{c}
\frac{V_{m}(s)}{s} \\
\frac{V_{s}(s)}{s}
\end{array}\right]^{*}
$$

Note that $\mathscr{Z}\left\{\frac{v}{s}\right\} \neq \mathscr{Z}\left\{\frac{1}{s}\right\} V(z)$. To be able to derive the transfer function from force to velocity we need to approximate $\mathscr{Z}\left\{\frac{v}{s}\right\}$ based on one of the three approximation methods (forward difference, backward difference and Tustin transformation) discussed in Section 3. In this paper it is assumed that $C_{m}=K_{m}+B_{m} s$ and $C_{s}=K_{s}+B_{S} s$. In agreement with $[9,10]$, to have the $z$ domain equivalent of $C_{m}$ and $C_{s}$, the backward difference is used to approximate $s$ :

$$
C_{m}(z)=K_{m}+\frac{B_{m}(z-1)}{T z} \quad C_{s}(z)=K_{s}+\frac{B_{s}(z-1)}{T z}
$$

Passive Terminations, No Delay Here, similar to HVE systems, it can be easily shown that the forward difference approximation method derives the worst-case condition for stability. Thus, for brevity the procedure resulting in choosing forward difference method is not included in this paper. Assuming that $t_{d}=0$, the force to velocity mapping will be:

$$
\left[\begin{array}{l}
F_{h}(z) \\
F_{e}(z)
\end{array}\right]=\left(\left[\begin{array}{cc}
b_{m} & 0 \\
0 & b_{s}
\end{array}\right]+H(z) \frac{T}{z-1}\right)\left[\begin{array}{l}
V_{m}(z) \\
V_{s}(z)
\end{array}\right]
$$

Again based on Theorem 2.1 and Definition 1 three conditions are to be satisfied. The first condition in Definition 1 requires all poles of $G^{-1}(z)$ to lie inside or on the unit circle, which obviously is the case here. As it can be clearly seen in (24), $z=1$ is a simple pole and in order to satisfy the second condition for positive realness the residue matrix corresponding to this pole must be positive semidefinite. For this pole the residue matrix is

$$
R_{0}=\left[\begin{array}{cc}
K_{m} T & -K_{m} T \\
-K_{s} T & K_{s} T
\end{array}\right]
$$

which is clearly positive semidefinite since $K_{m}, K_{s}, T>0$ and $\operatorname{det}\left(R_{0}\right)$ is zero. The only remaining condition is the second condition in Definition 1, which requires $G\left(e^{j \omega}\right)+G^{T}\left(e^{-j \omega}\right)$ to be positive semidefinite. Substituting $z=\cos (\omega T)+j \sin (\omega T)$, leading principle minors of $G\left(e^{j \omega}\right)+G^{T}\left(e^{-j \omega}\right)$ need to be positive semidefinite. Therefore,

$$
\begin{aligned}
& b_{m}-\frac{K_{m} T}{2}+B_{m} \cos (\omega T)>0 \\
& \operatorname{det}\left(G_{1}^{-1}\left(e^{j \omega T}\right)+G_{1}^{T-}\left(e^{-j \omega T}\right)\right)= \\
& \left(2 b_{m}-K_{m} T+2 B_{m} \cos (\omega T)\right)\left(2 b_{s}-K_{s} T+2 B_{s} \cos (\omega T)\right)- \\
& \left(\left(\frac{K_{m}+K_{s}}{2}\right) T-\left(B_{m}+B_{s}\right) \cos (\omega T)\right)^{2}- \\
& \left(\left(\frac{K_{m}-K_{s}}{2}\right) T \cot \left(\frac{\omega T}{2}\right)+\left(B_{m}-B_{s}\right) \sin (\omega T)\right)^{2}>0
\end{aligned}
$$

It can be easily shown that condition (27) is valid for $C_{m}=C_{s}=$ $K+\frac{B(z-1)}{T z}$ if

$$
\frac{b_{m} b_{s}}{b_{m}+b_{s}}>\frac{K T}{2}-B \cos (\omega T)
$$

Condition (28) is dependent on the frequency $\omega$ and since $\cos (\omega T)$ can vary between -1 and 1 , the worst-case for conditions (26) and (28), assuming $C_{m}=C_{s}=K+\frac{B(z-1)}{T z}$ and for $b=\min \left\{b_{m}, b_{s}\right\}$, a sufficient condition for absolute stability of a non-delayed sampleddata PEB bilateral teleoperation system will be

$$
b>K T+2 B
$$

Passive Terminations, Delay For a delayed sampled-data PEB bilateral teleoperation system, again the three approximation methods are compared and the worst-case condition is chosen as the absolute stability condition. For brevity, only the procedure using the forward approximation method is shown here because it returns the worst-case condition for the stability of the delayed sampled-data PEB bilateral teleoperation system. The resulting inverse transfer matrix will be

$$
\begin{aligned}
& G^{-1}(z)= \\
& {\left[\begin{array}{cc}
b_{m}+\left(\frac{K_{m} T}{z-1}+\frac{B_{m}}{z}\right) & -z^{-n}\left(\frac{K_{m} T}{z-1}+\frac{B_{m}}{z}\right) \\
-z^{-n}\left(\frac{K_{s} T}{z-1}+\frac{B_{s}}{z}\right) & b_{s}+\left(\frac{K_{s} T}{z-1}+\frac{B_{s}}{z}\right)
\end{array}\right]}
\end{aligned}
$$

Same as before, based on Definition 1, three conditions are to be satisfied. The first and third conditions are the case here since all poles of each element of $G^{-1}(z)$ lie inside or on the unit circle and 
the pole at $z=1$ is simple with the same residue matrix as (25), which is clearly positive semidefinite. The third condition which requires $G^{-1}\{j \omega\}+G^{-T}\{-j \omega\}$ to be positive semidefinite, is yet to be checked. Substituting $z=e^{j \omega T}=\cos (\omega T)+j \sin (\omega T)$, the second condition in Definition 1 for positive realness of $G^{-1}(z)$ will lead to the following conditions:

$$
\begin{gathered}
b_{m}+B_{m} \cos (\omega T)-\frac{K_{m} T}{2}>0 \\
\operatorname{det}\left(G\left(e^{j \omega T}\right)+G^{T}\left(e^{-j \omega T}\right)\right)= \\
4\left(b_{m}+B_{m} \cos (\omega T)-\frac{K_{m} T}{2}\right)\left(b_{s}+B_{s} \cos (\omega T)-\frac{K_{s} T}{2}\right)- \\
4\left(\frac{\left(K_{m}+K_{s}\right) T}{2}\left(\cos \left(\omega t_{d}\right)+S\right)-\left(B_{m}+B_{s}\right) \cos \left(\omega\left(T+t_{d}\right)\right)\right)^{2}- \\
4\left(\frac{\left(K_{m}-K_{s}\right) T}{2}(\sin (\omega T)-S)-\left(B_{m}-B_{s}\right) \sin \left(\omega\left(T+t_{d}\right)\right)\right)^{2}>0
\end{gathered}
$$

where $S=\frac{\sin \left(\omega t_{d}\right) \sin (\omega T)}{(1-\cos (\omega T))}$. Assuming that $t_{d} / T=n$ is an integer and for equal controllers $C_{m}=C_{s}=K+\frac{B(z-1)}{T z}$, the worst-case for (32) occurs when $S$ has its maximum value. Therefore, solving the $\frac{d}{d \omega} S=0$ will lead us to $\cos (\omega T)=1$, which is confirmed to give the maximum value of $S$ by checking the sign of the second derivative of $S$ when $\cos (\omega T)=1$. Then, it can be easily shown that condition (32) will simplify to

$$
\left(b_{m}+B-\frac{K}{2}\right)\left(b_{s}+B-\frac{K}{2}\right)-\left(\frac{K T}{2}+K t_{d}-B\right)^{2}>0
$$

With $b=\min \left\{b_{m}, b_{s}\right\}$, the absolute stability condition for a delayed sampled-data teleoperation system under passive terminations will be

$$
b+2 B>K T+K t_{d}
$$

\section{Simulation Study}

In this section, the conditions derived throughout the paper are tested using MATLAB/Simulink. In order to test conditions (13) and (18), the sampled-data HVE system in Figure $2 \mathrm{a}$ is simulated in MATLAB/ Simulink. To determine the stability of the system, the system outputs are monitored for boundedness at all times if any output goes unbounded, the system is unstable. The master robot has been modeled with $m=0.15$ and $b=0.1$. The operator model was considered to be $\frac{1}{s}$; note that $\mathfrak{R}\left\{\frac{1}{s}\right\}=0$ makes it a least-passive operator corresponding to a worst-case scenario for the coupled system stability. During the simulations, the sampling time is increased by steps of $1 \mathrm{~ms}$. For each sampling time, the controller gain $K$ is changed to find the largest gain value for which the system remains stable. In Figure 5, each of these maximum controller gain values at a given sampling period is represented by a blue star. Evidently, these simulation data points are very close to the solid blue line, which correspond to the theoretical borderline given by (13). Therefore, the simulations confirm the theoretical condition (13). For the delayed HVE system with $m=0.15$, $b=0.1$ and $B=0$, the delay $t_{d}$ is set to $10 T$ and again simulations are conducted with the same procedure as before. As shown in Figure 5, again the simulation data points represented by red stars are close to the theoretical borderline (18). Again, these simulation data points are very close to the solid red line (corresponding to the theoretical borderline given by (18)), confirming the theoretical condition. Also as shown in Figure 5, for the same passive operator, delay causes the stability region to shrink. This was predictable if one compares the theoretical conditions (13) and (18) for $B=0$. In

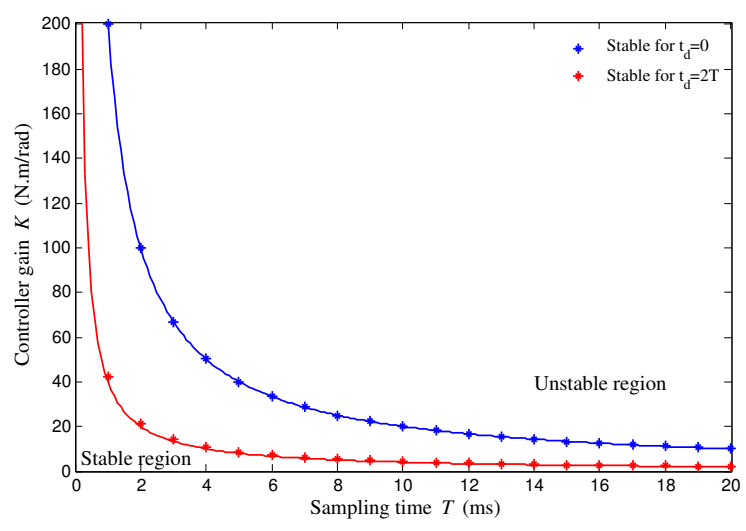

Figure 5: Simulation data points and corresponding theoretical borderlines for nondelayed (blue) and delayed (red) HVE system

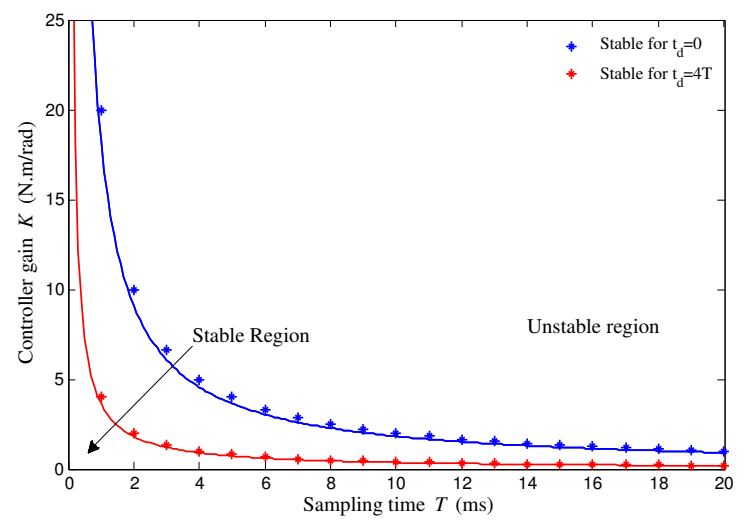

Figure 6: Simulation data points and corresponding theoretical borderlines for nondelayed (blue) and delayed (red) sampled-data PEB bilateral teleoperation system

order to test conditions (29) and (34), which correspond to passive terminations without and with delay, the sampled-data bilateral teleoperation system in Figure 4a is simulated in MATLAB/Simulink. Again, to determine the stability of the system, the system outputs are monitored for boundedness at all times. For the non-delayed bilateral teleoperation system with $m_{m}=m_{s}=0.015, b=0.02$ and $B=0$, simulations have been conducted. The operator and the environment models are considered to be $\frac{1}{s}$. Following a similar procedure as for the non-delayed HVE system, the controller gain $K$ is changed to find the largest gain value for which the system remains stable. As shown in Figure 6, the blue stars, represent each of these maximum controller gain values at a given sampling period. Evidently, these simulation data points are very close to the solid blue line, which correspond to the theoretical borderline given by (29). Simulations confirm the theoretical condition (29), since the simulation data points are very close to the solid line, which correspond to the theoretical borderline given by (29). Conducting similar simulations for the delayed bilateral teleoperation system with a delay set to $t_{d}=4 T$ as shown in Figure 6, again the simulation data points represented by red stars are very close to the solid red line which correspond to the theoretical borderline (34). Thus, simulations confirm the theoretical condition. Same as before, comparing the non-delayed (blue) and delayed (red) sampled-data systems, it is evident that delay causes the stability region to shrink, Figure 6. 


\section{CONCLUSION AND FUtURE WORK}

This paper studied the stability of both haptic virtual environment systems and PEB bilateral teleoperation systems based on the discrete-time circle criterion. The proposed stability analysis method enables a unified framework in which sampling time and communication time delay as two major destabilizing factors can be addressed. Simulation results confirm the validity of the proposed stability conditions.

\section{REFERENCES}

[1] O. Astley and V. Hayward. Design constraints for haptic surgery simulation. In IEEE International Conference on Robotics and Automation, 2000, 3: 2446-2451, 2000

[2] K. J. Astrom and B. Wittenmark. Adaptive Control. Addison-Wesley Longman Publishing Co., Inc., Boston, MA, USA, 2nd edition, 1994.

[3] C. Carignan and H. Krebs. Telerehabilitation robotics: bright lights, big future? Journal of Rehabilitation Research and Development, 43(5):695-710.

[4] J. Colgate and G. Schenkel. Passivity of a class of sampled-data systems: Application to haptic interfaces. Journal of Robotic Systems, 14(1):37-47, 1997

[5] J. Gil, A. Avello, A. Rubio, and J. Florez. Stability analysis of a 1 dof haptic interface using the routh-hurwitz criterion. IEEE Transactions on Control Systems Technology, 12(4):583-588, July 2004.

[6] J. Gil, S. Sanchez, T. Hulin, C. Preusche, and G. Hirzinger. Passivity and Stability Boundaries for Haptic Systems with Time Delay.
IEEE Transactions on Control Systems Technology, online submission, 2013. doi:10.1109/TCST.2013.2283372

[7] L. Hitz and B. D.Anderson. Discrete positive-real functions and their application to system stability. Proceedings of the Institution of Electrical and Engineers, 116(1):153-155, 1969.

[8] T. Hulin, C. Preusche, and G. Hirzinger. Stability boundary for haptic rendering: Influence of human operator. In IEEE International Con ference on Intelligent Robots and Systems, pages 3483-3488, 2008.

[9] A. Jazayeri and M. Tavakoli. A passivity criterion for sampleddata bilateral teleoperation systems. IEEE Transactions on Haptics, 6(3):363-369, 2012

[10] A. Jazayeri and M. Tavakoli. Absolute stability analysis of sampleddata scaled bilateral teleoperation systems. Control Engineering Practice, 21(8): 1053 - 1064, 2013.

[11] H. Khalil. Nonlinear Systems. Prentice Hall, 2002.

[12] D. S. P. Hokayem and M. Spong. Suboptimal master-slave teleoperation control with delays. In Proceedings of the 2006 American Control Conference, Minnesota, USA, June 2006

[13] J. Sheng and P. Liu. A review of bilateral sampled-data control of teleoperators. In IEEE International Conference on Robotics and Biomimetics385-390, Shenyang, China, Aug 2004.

[14] S. Ullrich and T. Kuhlen. Haptic palpation for medical simulation in virtual environments. IEEE Transactions on Visualization and Computer Graphics, 18(4):617-625, 2012. 